\title{
BATTLEFIELD EXPLOSIVE ORDNANCE DISPOSAL ROBOT
}

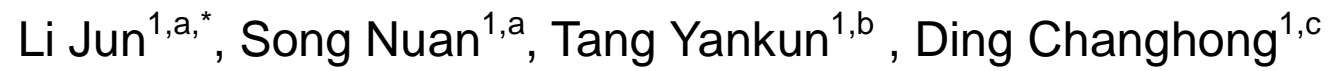 \\ ${ }^{1}$ Basic Course Department, Renmin Street 7855, Changchun, Jilin, 130000, China \\ achuanghu8521177@126.com, 'Laotang_506@163.com, 'dingchanghong777@163.com
}

\begin{abstract}
Key Words: Robot, Organization design, Control.
Abstract: With the Arduino microcontroller the battlefield EOD robot is composed of double body connecting with double joints, and all of the wheels composed of double track and vice round to carry and to facilitate its adapt to a variety of complex topography on the battlefield. Double joints can be very good to make robot joint surface, the robot can walk faster on complex terrain, climbing ability, and improve the stability. Gripping device of EOD robot is composed of three joints and six degrees of freedom mechanical arm. It can improve the flexibility of mechanical arm, also can increase the object transfer, object of obstacles to avoid flexibility. This project can be used in a variety of complex battlefield environment, for explosives can adopt many ways such as investigation, elimination, transfer to exclude danger.
\end{abstract}

\section{Introduction}

In future war, the battlefield environment is variable and full of unknown. We can use the robots instead of people as much as possible in order to reduce personnel loss. So we need the robots that can adapt to a variety of terrain, mobile fast and have high climbing ability. The occurrence of war is more and more concentrated in the local and the city. The complex terrain of the city, many combatants can't reach region. It becomes dangerous. In the world, there are a lot of countries developing EOD robots instead of workers in complex areas. Their research are also various, such as mobile carrier mobility, the reasonable degree of freedom mechanical arm configuration, quickness and effectiveness of the use of robots, operation safety, psychological tension reducing of EOD personnel and the intensity of labor, etc. Foreign EOD robot industry has already formed the scale, many famous manufacturers to develop new products [1]. Our Chinese also has several universities and research institutions in research EOD robot, and obtained a series of research results. But they are for civilian use. There is no EOD robot designed for the battlefield.

This project combined with the practical application, the analysis of modern robotics, determine the design goal. It is finished with 6-DOF manipulator. And considering the complex battlefield terrain, it has more excellent performance adopted the unique design of caterpillar vehicles ditch climbs.

\section{Mechanical Structure Design}

This project is composed of caterpillar vehicles and the mechanical arm. Caterpillar vehicles are adopted by rigid good quality aluminum alloy, and designed with the crawler, triangle wheel as a transmission mechanism. Its cross-country performance is very good. Mechanical arm is composed of base, four u-shaped bracket and an object can be clamping clip. Their activities depend on six steering gear control. And we can operate flexibly.

\section{Design of Caterpillar Vehicles}

About the mobile mechanism of EOD robot mainly include: wheel, caterpillar and joint type three kind of walking robot [1]. They have different advantages and disadvantages. Considering 
above the battlefield environment, the goal of this project is vehicle bodywork that can complete a variety of complex terrain such as trenches, crater, hills and steps. So we design considering of three points.

A. Double car body structure. We adopt double car body structure and use two single joints connection so it is increased for joint sex ground, all caterpillar fulcrum force, as well as the ability to travel.

B. The track structure. Track structure is adopted for each body. Caterpillar can reduce the pressure of unit area, and on the requirements can be smaller. And the quality of the defense and cross-country performance is better than wheel robot. Also it has better ability to through the trenches and the vertical wall. Caterpillar can firmly seize the ground and will not slip such as rain, snow, ice or uphill road because of the segments with decorative pattern.

C. Deputy vice wheel. Vice wheel adopts triangle structure. With a certain angle and vice round the wheel can rotate up and down with the $\mathrm{X}$ axis in -10 degrees to 45 degrees. The ability of climbing is more increased. We can have fixed effects on the caterpillar body like a hook when climbing the slope steep.

All-terrain vehicles bring design idea. Joining vice round is the most difficult in our production. How to select the angle? How to solve the problem of vice round limit in the case of purely physical way? How to rotate the vice round under non-contact control? We solved all of above problems. This design is verified by experiments. The caterpillar fit the ground environment in the well. It is operated convenient and nimble. It can be provide a lot of thrust by four motors power. In the case of a front wheel dangling it can still rely on the rear wheels provide thrust forward. Caterpillar vehicles physical objects as shown in figure. 1 .

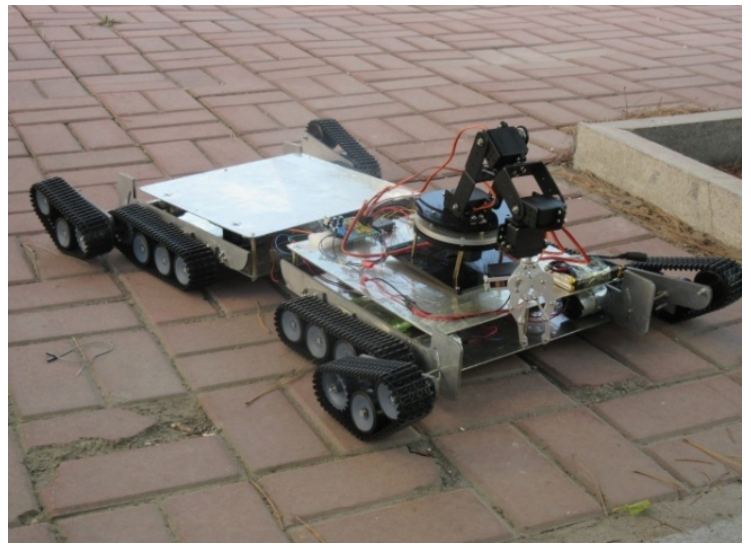

Figure. 1. physical caterpillar vehicles

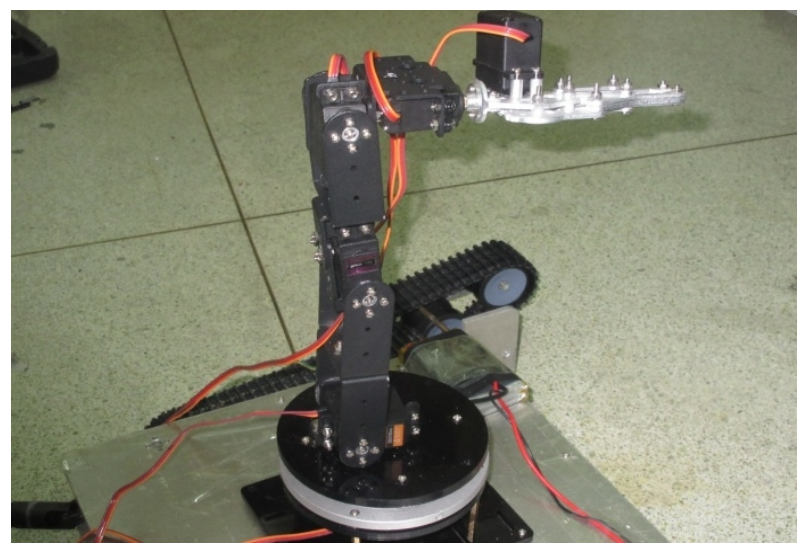

Figure. 2. physical mechanical arm

\section{Design of Mechanical Arm}

In this project the mechanical arm can grab medium and small explosives into the cartridge. And then the explosives are detonated in blasting barrels or transferring cartridge to safety and set off. Physical objects as shown in figure. 2, the total length of the mechanical arm is about $400 \mathrm{~mm}$. It is controlled by the Arduino board. And we can non-contact operate the mechanical arm equipped with remote control realize.

How to make the mechanical arm safely, quickly and easily autonomous or semi-autonomous fetching explosives is an important research content of EOD operation. Basically all the EOD robot arm have at least three rotational degrees of freedom, the big arm turning around mobile carrier, forearm rotation around the big arm and wrist turning. Mechanical arm can be thought of as composed of a series of joints connecting rod. This item is made of six degrees of freedom mechanical arm. It has a wide working space. We have adopted five joints and a rotating base. Each 
joint can move within a certain range. The base can rotate 360 degrees. Power system is provided by six steering gears who is a kind of flexible operation of mechanical and electrical equipment. We can achieve the object of activity angle adjustment through steering gears. The control of this project is very good implementation relied on the six steering gears.

\section{Control System Design}

Aiming at the objective of the mechanical structure, design of control system is shown in figure. 3.

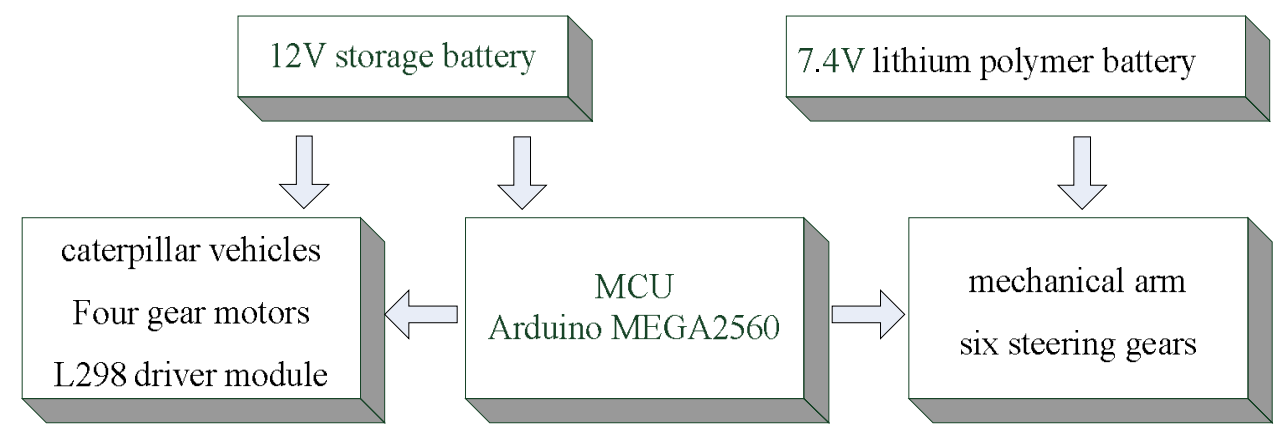

Figure. 3. system block diagram

\section{Master Control Module}

The objective of the master control module is the Arduino Mega 2560 development board. It has a low price, simple programming environment, open source extensible software and hardware. It is used very convenient.

\section{Mechanical Arm Control Module}

In the small robot control system, the vast majority steering gears are used to control the movement of the arm at present. The steering gear is a kind of position (angle) of the servo drive, very suitable for the system who need angle changing and continuous controlling. Arduino who can output the PWM signal, provides the steering gear rotating to the target location at full speed. When it arrived at the target location, it will keep the position automatically. We use the digital servo MG996R and DS3115 with better performance. It can external large rotation in loading motion, output larger torque and have great shaking resistance. And its internal potentiometer is high linearity when the gear come to limit position will not deviate from the goal.

\section{Caterpillar Vehicles Control Module}

Considering the load condition of caterpillar vehicles and speed requirements, respectively on two bodies mount two $12 \mathrm{~V}$ DC and $7500 \mathrm{r} / \mathrm{min}$ gear motors. Microcontroller output current only dozens of mA. It can't drive motor, so between the motor and single chip microcomputer to join special L298 motor driver module. The main body of the module is 15 pin Multiwatt package L298N chip. Chip internal contains 4 channel logical drive circuit, can be driven by two DC motors, or a two phase stepper motor. Its output voltage is up to $50 \mathrm{~V}$. In this project, by programming control on the left side of the two wheels or on the right side of two wheels require to reach the same speed, therefore adopted four L298 module to drive four motors respectively.

\section{Wireless Remote Control Module}

EOD operation is very dangerous. We use the PS2 game controller for remote control operation. PS2 joystick control on the handle of caterpillar vehicles move forward, backward, turn left, turn right, stop. Six buttons control the rotation of the steering gear, which can be easily implemented to control the mechanical arm to grab and place. 


\section{Power Supply Module}

The power supply to the robot should be needed such as long distance, mobility and flexibility movement, etc. We used the storage battery as the main power supply, to the Arduino microcontroller and four DC gear motor power supply. Due to the working characteristics of the steering gear, we choose the discharge rate of lithium polymer battery as the auxiliary power supply for six steering gears.

\section{Summary}

This project has practical application value. The EOD robot based on the Arduino can adapt to most of the complex battlefield terrain. It can climb the steps of the vertical for short also. With independent design of caterpillar vehicles it can range in slopes, sand and other complex terrain. 6 degree of freedom mechanical arm can remote operation smoothly fetching explosive. However this project has a lot of shortcomings, such as low intelligent degree, slow operation speed. We need to do further research.

\section{References}

[1] Zeng Jianjun. Research on teleoperation performance of explosive ordnance disposal robot. Shanghai Jiao Tong university, Ph.D. Dissertation, 2010: 8-9, 44-45.

[2] Liu Yan, Zhang Cong, Wang Chensheng. The path planning algorithm based on six degrees of freedom mechanical arm system [J]. Journal of construction machinery, 2013.7: 89-92.

[3] Cheng Chen. Arduino practical guide [M]. Mechanical industry publishing house, 2012.

[4] Cai Zixing. Robotics. [M]. Tsinghua university press, 2000. 